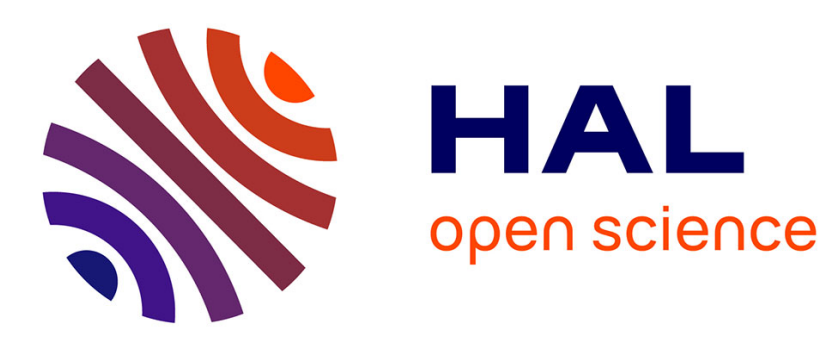

\title{
Needle Segmentation in 3D Ultrasound Volumes Based on Machine Learning for Needle Steering
}

Guillaume Lapouge, Hatem Younes, Philippe Poignet, Sandrine Voros, Jocelyne Troccaz

\section{To cite this version:}

Guillaume Lapouge, Hatem Younes, Philippe Poignet, Sandrine Voros, Jocelyne Troccaz. Needle Segmentation in 3D Ultrasound Volumes Based on Machine Learning for Needle Steering. Hamlyn Symposium on Medical Robotics, Jun 2019, Londres, United Kingdom. hal-02276986

\section{HAL Id: hal-02276986 https://hal.science/hal-02276986}

Submitted on 3 Sep 2019

HAL is a multi-disciplinary open access archive for the deposit and dissemination of scientific research documents, whether they are published or not. The documents may come from teaching and research institutions in France or abroad, or from public or private research centers.
L'archive ouverte pluridisciplinaire HAL, est destinée au dépôt et à la diffusion de documents scientifiques de niveau recherche, publiés ou non, émanant des établissements d'enseignement et de recherche français ou étrangers, des laboratoires publics ou privés. 


\title{
Needle Segmentation in 3D Ultrasound Volumes Based on Machine Learning for Needle Steering
}

\author{
G. Lapouge ${ }^{1,2}$, H. Younes ${ }^{1}$, P. Poignet ${ }^{2}$, S. Voros ${ }^{1}$, J. Troccaz ${ }^{1}$ \\ 1 Univ. Grenoble Alpes, CNRS, Grenoble INP, TIMC-IMAG, F-38000 Grenoble, France \\ 2Univ. Montpellier, CNRS, LIRMM, F-34090 Montpellier, France \\ \{hatem.younes ; guillaume.lapouge\}@univ-grenoble-alpes.fr
}

\section{INTRODUCTION}

To achieve needle steering and guide a flexible needle, it is necessary to localize it first. In the case of $3 \mathrm{D}$ ultrasound (US) in B-mode, the poor imaging quality and artefacts make it difficult to determine the needle pose. Needle localization issues specific to needle steering are reviewed in [1]. In [2], we proposed an observer of the needle tip pose. In [3], we detailed a machine learning approach for needle localization in 3D US volumes. In this paper, we describe the interconnection of both methods for precise curved needle localization in the context of needle steering.

\section{MATERIALS AND METHODS}

\section{Approach}

3D US compatible needle localization algorithms are reviewed in [4]. Localizing the needle in the whole US volume is computationally expensive. To manage realtime localization, we propose the interconnection of an observer that automatically provides a volume of interest (VOI), and a segmentation algorithm. Because the needle visibility in 3D US volume changes during the insertion, we propose machine learning to select adaptively needle voxels among the VOI voxels.

\section{Needle Pose Estimation}

The needle tip behavior is modeled by a unicycle kinematic model. The tip follows a circular path when it is inserted without rotation. In this work, we consider that the needle shaft follows the path of the needle tip. In this hypothesis, segmenting the needle tip over time is equivalent to segmenting the whole needle shaft. This hypothesis is validated experimentally.

The needle tip position and orientation are estimated in real time by the multi-rate unscented Kalman filter (MUKF) described in [2]. It takes as inputs the robot control commands and the measurement coming from the robot's sensors, needle tip segmentation results in the 3D US volumes and stiffness information of the surrounding tissue. The estimated path traced by the needle tip $\widehat{\Phi}$ defines a tubular volume of interest, of radius set by the user, for needle segmentation.

If the needle cannot be segmented in the ultrasound volume, the estimation continues until the needle becomes visible again. The segmentation results are then considered as measurements to update the estimation.

This work was partly supported by the Investissements d'Avenir programme (Labex CAMI, ANR-11-LABX0004) and by the FOCUS project (ANR-16-CE19-0011).

\section{Naïve Bayes classification}

As explained in [3], the VOI histogram can be modeled using an additive Gaussian Mixture Model (GMM):

$$
\mathrm{P}(\mathrm{I}(\mathrm{v}))=\sum_{\mathrm{i}=1}^{M} \pi_{\mathrm{v}_{\mathrm{i}}} \mathrm{P}_{\mathrm{i}}(\mathrm{I}(\mathrm{v}))
$$

Where $\mathrm{v}$ is a voxel and $\mathrm{I}(\mathrm{v})$ its intensity value, $\mathrm{M}$ is the number of mixed components, $\pi_{V_{i}}$ represents the coefficient of the $i^{\text {th }}$ component and $P_{i}$ is the Gaussian probability density function that defines the $\mathrm{i}^{\text {th }}$ component. Here we consider two classes $(\mathrm{i}=2)$ :

$$
\mathrm{Cl}=\left\{\text { Needle }\left(\mathrm{X}_{\mathrm{n}}\right) \text {, Background }\left(\mathrm{X}_{\mathrm{b}}\right)\right\} \text {. }
$$

Given the observed feature I(v), the Bayesian classifier assigns the most likely class as next equation:

$$
\mathrm{C}_{\text {Bayes }}(\mathrm{I}(\mathrm{v}))=\arg \max _{\mathrm{v}_{\mathrm{i}}} \mathrm{P}\left(\mathrm{Cl}=\mathrm{X}_{\mathrm{i}} \mid \mathrm{I}(\mathrm{v})\right) \text {. }
$$

In the case of binary classification, Bayesian classifier is summarized by the followed inequalities:

$$
\begin{aligned}
& \mathrm{X}_{\mathrm{n}} \\
& \pi_{x_{n}} \mathrm{P}\left(\mathrm{I}(\mathrm{v}) \mid \mathrm{Cl}=\mathrm{X}_{\mathrm{n}}\right) \underset{\mathrm{X}_{\mathrm{b}}}{\gtrless} \pi_{\mathrm{x}_{\mathrm{b}}} \mathrm{P}\left(\mathrm{I}(\mathrm{v}) \mid \mathrm{Cl}=\mathrm{X}_{\mathrm{b}}\right) .
\end{aligned}
$$

Each voxel of intensity $I(v)$, is classified as a needle member if the posterior probability of $\mathrm{X}_{\mathrm{n}}$ is larger than that of $\mathrm{X}_{\mathrm{b}}$. Several parameters are required to establish this probabilistic model. They vary from GMM to another, even in the same image. Though, each needle localization starts with the prediction of the required parameters.

\section{Expectation Maximization algorithm (EM)}

The unknown parameters representing the GMM are estimated by an EM algorithm. This iterative process computes the maximum likelihood of an observed feature for each iteration. The following equations represent the main instructions done iteratively to estimate the parameters.

$$
\begin{aligned}
\pi_{X_{i}}^{\text {new }} & =\frac{1}{N} \sum_{j=1}^{N} P\left(X_{i} \mid v_{j}, \theta^{k}\right) \\
\mu_{X_{i}}^{\text {new }} & =\frac{\sum_{j=1}^{N} v_{j} P\left(X_{i} \mid v_{j}, \theta^{k}\right)}{\sum_{j=1}^{N} P\left(X_{i} \mid v_{j}, \theta^{k}\right)} \\
\sigma_{X_{i}}^{\text {new }} & =\frac{\sum_{j=1}^{N} v_{j} P\left(X_{i} \mid v_{j}, \theta^{k}\right)\left(v_{j}-\mu_{X_{i}}^{n e w}\right)^{2}}{\sum_{j=1}^{N} P\left(X_{i} \mid v_{j}, \theta^{k}\right)}
\end{aligned}
$$

Where $\mathrm{N}$ is the number of voxels and $\theta^{\mathrm{k}}$ is the parameters vector to be estimated at the $\mathrm{k}^{\text {th }}$ iteration. 

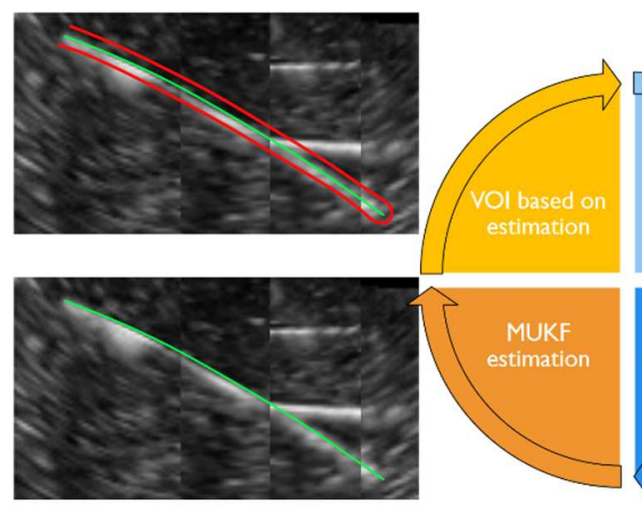

Fig. 1 Needle segmentation p

Starting from an initial set of values for $\theta$, the algorithm
uses the newly derived parameters as the guess for the next iteration. The iteration stops when the parameters values stabilize: $\left|\theta^{\mathrm{k}}-\theta^{\mathrm{k}+1}\right|<\varepsilon$. A meaningful initialization of $\theta$ is necessary for suitable convergence. More details can be found in [3].

\section{Needle tip segmentation}

The needle visibility depends on its location in the US volume. Before applying Bayesian classification, the VOI is cropped to keep the $40 \%$ of its original length which contain the needle extremity. In this way, we ensure that the tip and shaft voxel intensities are similar (i.e. can be considered as belonging to the same class $\mathrm{Xn})$.

The needle tip search is then done in a spherical neighborhood around the estimated needle tip. In that neighborhood, if there is no voxel belonging to $\mathrm{Xn}$, the segmentation fails. Else, the segmented tip is computed as the weighted centroid of the voxels in $\mathrm{Xn}$. The weighting $w_{p}$ of a voxel p verifies:

$$
w_{p} \propto w_{p_{1}} x_{t}-w_{p_{2}}\left(y-y_{0}\right)
$$

Where $w_{p_{1}}, w_{p_{2}} \in \mathbb{R}_{+}^{2}$ are set by the user (resp. 3 and 1 here); $x_{t}$ is the abscissa of the voxel in the estimated tip frame $\mathrm{F}_{\mathrm{t}}$ (see Fig. 1); $y$ (resp. $y_{0}$ ) is the ordinate of the voxel (resp. estimated tip) in the image frame F (see Fig. 1 ). The needle tip is therefore researched preferentially in the direction of the insertion $\left(x_{t}\right)$, and in the opposite direction of expected tip reverberation artefacts $(-y)$.

\section{RESULTS}

The proposed solution has been implemented in the context of automated needle insertions to reach a target. A beveled-tip 24 Gauge Nitinol needle was inserted and rotated into gelatin phantoms and pork tenderloin with a robot. The needle surface was either coated with polyurethane foam or etched with a laser. The ultrasound volumes were acquired at $1 \mathrm{~Hz}$ with a $3 \mathrm{D}$ end-fire probe 4DEC-9/10 used with the Ultrasonix Sonix RP ultrasound system. The US volume voxels are cubes with $0.4 \mathrm{~mm}$ edges. Running on an Intel ${ }^{\circledR}$ Core $^{\mathrm{TM}} \mathrm{I} 5-8300 \mathrm{H}$ CPU, an iteration of the algorithm takes between $20 \mathrm{~ms}$ and $30 \mathrm{~ms}$ depending on the VOI size.

60 manual needle tip segmentation $(0.3 \mathrm{~mm}$ repeatability) have been done by a non-expert individual among 4 different needle insertions ( 2 in pork tenderloin and 2 in gelatin phantoms). The 3D segmentation error $\Delta$ was computed as the Euclidean distance between manual and automatic tip segmentation. Axial segmentation

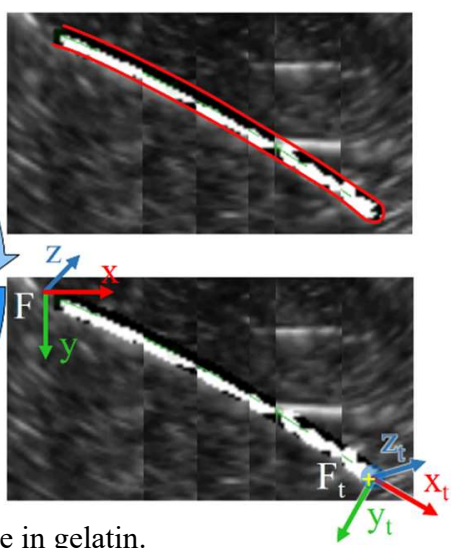

errors were computed as the Euclidean distance on each axis between manual and automatic tip segmentation. Results are reported in Table 1.

\begin{tabular}{|c|c|c|c|c|}
\hline Difference & $\Delta \mathrm{x}$ & $\Delta \mathrm{y}$ & $\Delta \mathrm{z}$ & $\Delta$ \\
\hline \multirow{2}{*}{ Gelatin } & $0.5 \pm 0.3$ & $0.2 \pm 0.2$ & $0.5 \pm 0.3$ & $0.8 \pm 0.3$ \\
& {$[0.0 ; 1.2]$} & {$[0.0 ; 0.8]$} & {$[0.0 ; 1.2]$} & {$[0.1 ; 1.5]$} \\
\hline \multirow{2}{*}{ Pork } & $0.3 \pm 0.2$ & $0.3 \pm 0.2$ & $0.4 \pm 0.3$ & $0.5 \pm 0.2$ \\
& {$[0.0 ; 0.5]$} & {$[0.0 ; 0.8]$} & {$[0.0 ; 0.6]$} & {$[0.2 ; 1.0]$} \\
\hline
\end{tabular}

Table 1 Difference between manual and automatic needle segmentation (mm). Format: mean \pm std [min;max].

The mean segmentation error in each direction is generally inferior to the spatial resolution of the probe. The results are therefore very close to voxel size precision and satisfactory for needle tip segmentation. Needle segmentation is more precise in pork than in gelatin. Indeed, in gelatin, the needle can be seen with a better contrast but suffers from greater reverberation artefacts than in pork.

Momentary needle loss occurred in 2 of the pork insertions with no significant impact on automatic segmentation error when the needle becomes visible again.

\section{DISCUSSION}

We have proposed an automated needle localization in 3D ultrasound volumes. The solution is composed of interconnected observer and segmentation algorithms. The solution is fully automatic and adapts to the conditions of insertions (i.e. different needles or insertion media). This interconnection allows for precise tip localization despite changing needle visibility in noisy 3D US volumes. It is also robust to momentary needle loss. This makes it suitable for clinically compatible needle steering.

\section{REFERENCES}

[1] C. Rossa, M. Tavakoli. Issues in closed-loop needle steering, Control Engineering Practice. May 2017; 62: 55-69.

[2] G. Lapouge, J. Troccaz, P. Poignet. Multi-rate unscented Kalman filtering for pose and curvature estimation in 3D ultrasound-guided needle steering. Control Engineering Practice. Nov. 2018; 80: 116-124.

[3] H. Younes, S. Voros S, J. Troccaz. Automatic needle localization in 3D ultrasound images for brachytherapy. IEEE 15th International Symposium on Biomedical Imaging (ISBI 2018). 2018; 1203-1207.

[4] Y. Zhao, Y. Shen, A. Bernard, C. Cachard, H. Liebgott. Evaluation and comparison of current biopsy needle localization and tracking methods using 3D ultrasound. Ultrasonics. Janv. 2017; 73: 206-220. 